\title{
Thyroid hormone controls the gene expression of HSV-1 LAT and ICPO in neuronal cells
}

\author{
Gautam R Bedadala ${ }^{1}$, Rajeswara C Pinnoji ${ }^{1}$, Jayavardhana R Palem ${ }^{1}$, Shao-Chung V Hsia ${ }^{1}$ \\ ${ }^{I}$ Department of Basic Pharmaceutical Sciences, University of Louisiana at Monroe College of Pharmacy, Monroe, LA 71209, USA
}

\begin{abstract}
Various factors/pathways including hormonal regulation have been suggested to control herpes simplex virus type 1 (HSV-1) latency and reactivation. Our computer analysis identified a DNA repeat containing thyroid hormoneresponsive elements (TRE) in the regulatory region of HSV-1 latency-associated transcript (LAT). Thyroid hormone (triiodothyronine, $T_{3}$ ) functions via its receptor TR (thyroid hormone receptor), a transcription factor. Present study investigated the roles of TR and $T_{3}$ in HSV-1 gene expression using cultured neuoroblastoma cell lines. We demonstrated that liganded TR activated LAT transcription, but repressed infected cell protein no. 0 (ICP0) transcription in the presence of LAT TRE. Chromatin immunoprecipitation (ChIP) assays showed that TRs were recruited to LAT TREs independently of $\mathrm{T}_{3}$ and hyperacetylated $\mathrm{H} 4$ was associated with the LAT promoter that was transcriptionally active. In addition, ChIP results showed that the chromatin insulator protein CCCTC-binding factor was enriched at the LAT TREs in the presence of TR and $T_{3}$. In addition, the BRG1 chromatin remodeling complex is found to participate in the $T_{3}$ /TR-mediated LAT activation since overexpression of BRG1 enhanced the LAT transcription and the dominant-negative mutant $\mathrm{K} 785 \mathrm{R}$ abolished the activation. This is the first report revealing that TR elicits epigenetic regulation on HSV-1 ICPO expression in neuronal cells and could have a role in the complex processes of HSV-1 latency/reactivation.
\end{abstract}

Keywords: HSV-1, thyroid hormone, chromatin, transcription, LAT, ICP0, latency

Cell Research (2010) 20:587-598. doi:10.1038/cr.2010.50; published online 13 April 2010

\section{Introduction}

Herpes simplex virus type-1 (HSV-1) causes diseases ranging from mild oral lesions to severe keratitis and lethal encephalitis. HSV-1 infection is composed of four phases: acute infection, establishment of latency, maintenance of latency, and reactivation from latency. During acute infection, HSV-1 targets epithelial cells where the virus undergoes active gene expression in a cascade fashion and generates virion progeny after replication. The expression cascade is characterized by a sequential order with genes grouped into three categories: immediate-

\footnotetext{
Correspondence: Shaochung Hsia

Tel: +318-342-1747; Fax: +318-342-1737

E-mail: hsia@ulm.edu

Abbreviations: HSV-1 (herpes simplex virus type 1); IE gene (immediateearly gene); ICP0 (infected cell protein no. 0); HDACs (histone deacetylase complexes); LAT (latency-associated transcript); ChIP (chromatin immunoprecipitation); CTCF (CCCTC-binding factor); BRG1 (brahmarelated gene 1); hpi (hours postinfection)

Received 13 July 2009; revised 26 Octorber 2009; accepted 30 November 2009, published online 13 April 2010
}

early (IE), early (E), and late (L). After the completion of replication and egress, acute infection may be followed by the establishment of latency in the innervating neurons of the peripheral nervous system. In contrast to the acute infection, during latency viral transcriptions are largely void except in one region, which codes for the latency-associated transcript (LAT) [1-4]. The latent virus can survive in the neurons throughout the life of the host and may reactivate in response to various stimuli after the reversal of viral gene silencing [3, 5-8]. A variety of mechanisms have been proposed to describe the establishment of viral latency and reactivation such as altered immune response [9-12], microRNAs-induced gene silencing [13], differential neuronal suppression [14-17], hormonal regulation [18-21], and repressive chromatin [22-28]. Our laboratory identified thyroid hormoneresponsive elements (TREs) in several HSV-1 regulatory regions and one of them is located in the LAT regulatory sequence, suggesting that thyroid hormone (triiodothyronine, $\mathrm{T}_{3}$ ) could have roles in the transcription of LAT and neighboring regions and therefore modulate the viral latency and reactivation. 
TRE is the binding site of transcription factor thyroid hormone receptors (TRs). The regulatory activity of TR is dependent on the ligand thyroid hormone. Thyroid hormone is produced by the addition of iodine to tyrosine in the thyroglobulin protein [29]. The primary hormone product is Thyroxine $\left(\mathrm{T}_{4}\right)$, but it is converted to the potent $T_{3}$ in target tissues [30]. $T_{3}$ interacts with TRs to elicit its biological effects [31]. TRs belong to the nuclear hormone receptor superfamily, and vertebrates have two TR genes, TR $\alpha$ and TR $\beta$ [32]. The TRE-TR interaction is, nonetheless, independent of $\mathrm{T}_{3}$, implicating that both unliganded as well as liganded TRs participate in the regulation of gene expression [33].

The present study analyzes the interrelationship of $\mathrm{T}_{3}$, TRs, and chromatin in HSV-1 gene regulation in neuronal cells. The gene regulation of LAT and infected cell protein no. 0 (ICP0) was investigated in mouse neuroblastoma cells N2a and N2aTR $\beta$, in which the TR $\beta$ is constitutively expressed. The effects of TR and $T_{3}$ on histone modification and cofactor recruitment to the promoters were also assessed. Our results showed that TR can either activate or repress these genes dependent on the presence of $T_{3}$ via recruitment of various co-activators or corepressors for distinct histone modifications and chromatin remodeling.

\section{Results}

\section{Location of HSV-1 LAT TRES}

We identified putative TREs close to the LAT regulatory region in the HSV-1 genome (Figure 1A). The first sets of TREs were found in the terminal repeat long region from 9038 to 9208 , coexisting with the reiteration set-1 (RE-1) (Figure 1A). The second sets were located from 117164 to 117 334, overlapping with the internal repeat long RE-1 (Figure 1A). The positive TRE sequence 5'-GGG AGA-3' was repeated 21 times in these regions, separated by one or four nucleotides (Figure 1B). The TRE is $1.4 \mathrm{~kb}$ upstream of the LAT TATA box. We hypothesize that TR may bind to this TRE and regulate the transcription of LAT and the neighboring gene ICP0.

A

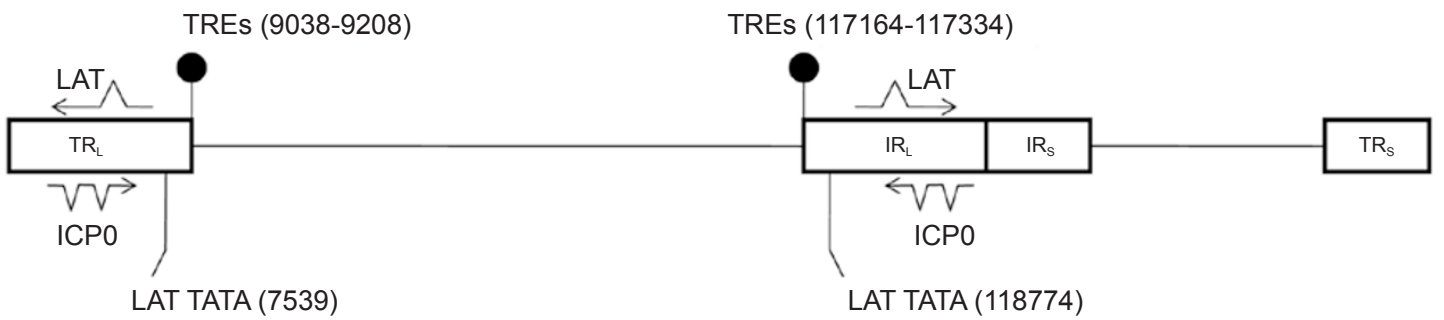

B

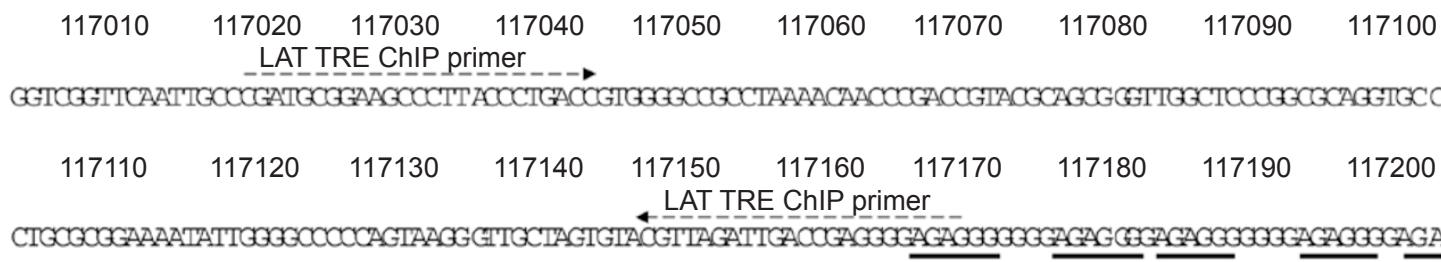

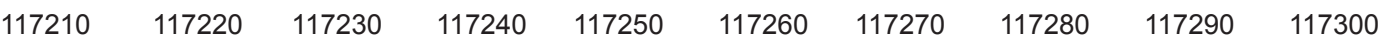

GGGGGGCACAGGGCAGAGGGGGGGAGAGGGGA GAGGGGGGCAGAGGGCAGAGGGGGGGAGAGGGCAGAGGGGGGZAGAGGGGAGAGGGGGGCAGAGGGGA

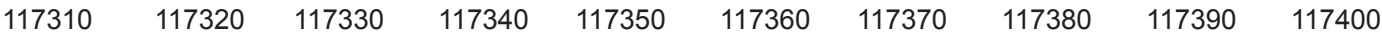

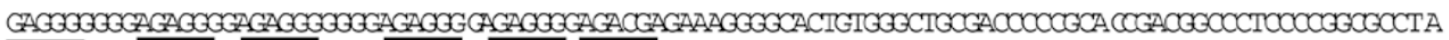

Figure 1 HSV-1 LAT TREs. (A) Two sets of positive TREs were identified in HSV-1 genome within LAT regulatory region. The first set was found in the terminal repeat long $\left(T R_{L}\right)$ reiteration set 1 and the second set was located in the internal repeat long $\left(I R_{L}\right)$ reiteration set 1. (B) The positive TRE sequence 5'-GGG AGA-3' was repeated 21 times in these regions separated by one or four nucleotides. These TREs are $\sim 1.4 \mathrm{~kb}$ upstream of the LAT TATA box. The figure is based on HSV-1 complete genome sequence (Accession no. X14112). LAT TRE ChIP primers were designed adjacent to one end. 
Liganded TR positively regulated LAT but repressed $I C P 0$ transcription

The regulatory effect of TR on LAT and ICP0 was examined by transfecting N2a or N2aTR $\beta$ cells with plasmid pSG28, which contains ICP0 and LAT including LAT TREs. RT-PCR assays showed that LAT transcription was up-regulated by TR and $\mathrm{T}_{3}$ (Figure 2A). ICP0, however, was down-regulated by liganded TR (Figure 2A). To address whether or not ICP0 was directly regulated by TR and $\mathrm{T}_{3}$, transfections were performed using plasmid pAA3, which contains the ICP0 promoter but not LAT TREs. The results showed no regulation on ICP0 transcription (Figure 2B, compare lanes 4 and 5). Effect of TR on LAT was also investigated, and the RTPCR assays revealed that liganded TR did not regulate LAT transcription in the absence of LAT TRE (Figure 2B, compare lanes 8 and 9). Transfection of phMGFP followed by fluorescent microscopy revealed similar numbers of cells emitting green fluorescence, suggesting that transfection efficiency was similar between these two cell types (data not shown). Together, these observations indicated that LAT was positively regulated by TR and $\mathrm{T}_{3}$, while ICP0 was repressed by liganded TR, and this TR-mediated repression requires LAT TREs.

Viral infections were performed to confirm the observation from the transfection experiments. Strain $17 \mathrm{Syn}^{+}$ was used at MOI $=10$ to infect N2a and N2aTR $\beta$ cells with or without $100 \mathrm{nM} \mathrm{T}$ followed by RNA isolation and RT-PCR assays. The results showed no TR-mediated regulatory effect on LAT at $8 \mathrm{~h}$ postinfection (hpi), probably due to high MOI and IE transactivation (data not shown). After the addition of $50 \mu \mathrm{g} / \mathrm{ml}$ of CHX during infection to inhibit IE protein synthesis, LAT transcription was up-regulated by liganded TR at 8 hpi (Figure $3 \mathrm{~A}$ ). TR and $\mathrm{T}_{3}$, at the same condition, caused a $60 \%$ reduction on ICP0 transcription, as measured by Syngene's molecular biology software GeneTools (Syngene, Frederick, MD, USA) (Figure 3B and 3C). These results dem-

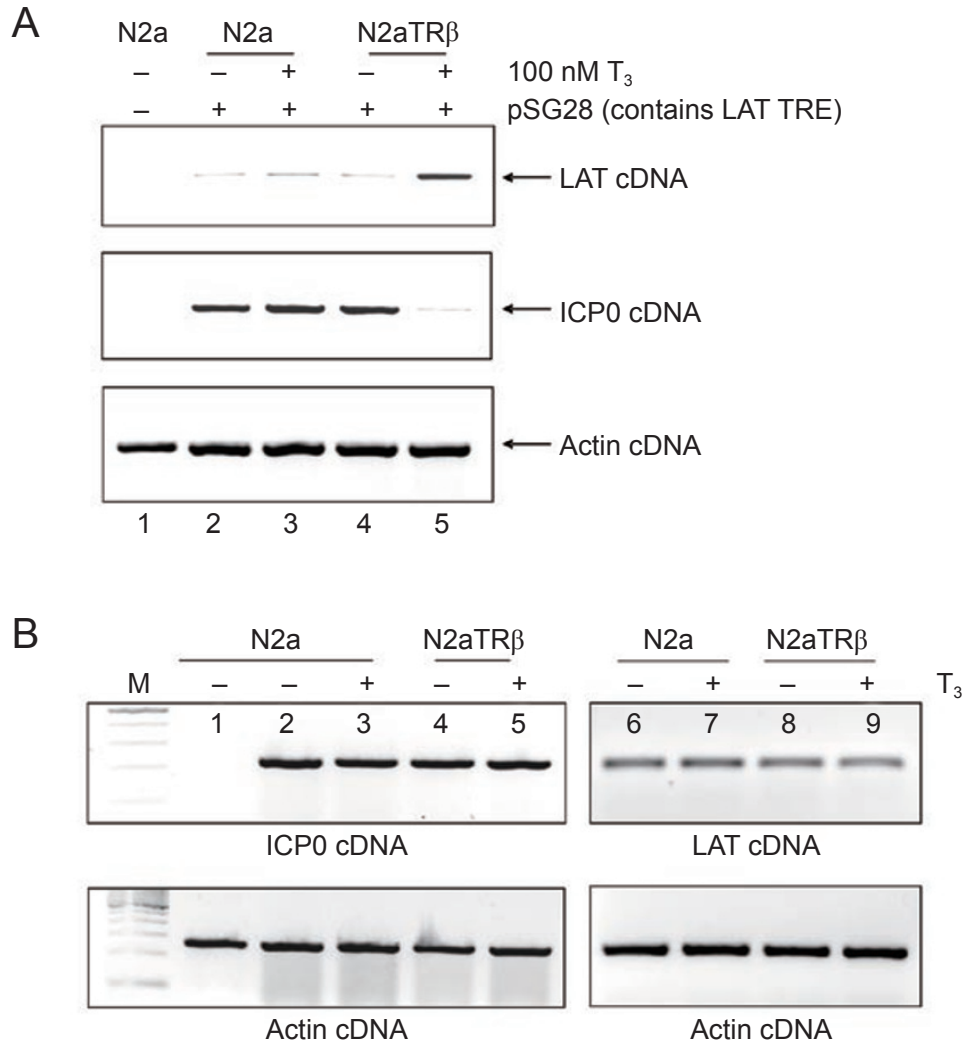

Figure 2 TR/T 3 -mediated regulation on HSV-1 LAT and ICP0. (A) N2a and N2aTR $\beta$ cells were transfected with plasmid containing intact LAT TRE, LAT, and ICPO to analyze the TR-mediated regulatory effect. Total RNA was purified after $48 \mathrm{~h}$ and subjected to RT-PCR assays using primers against LAT and ICPO. Actin primers were used as control. It showed that liganded TR up-regulated LAT but down-regulated ICPO (compare lanes 4 and 5). (B) Transfection of N2a and N2aTR $\beta$ cells with plasmids without LAT TREs but with complete ICPO gene. Total RNA was purified and subjected to RT-PCR assays using primers against LAT and ICP0. Actin primers were used as control. Results showed that neither ICPO nor LAT was regulated in the absence of LAT TRE. 
A

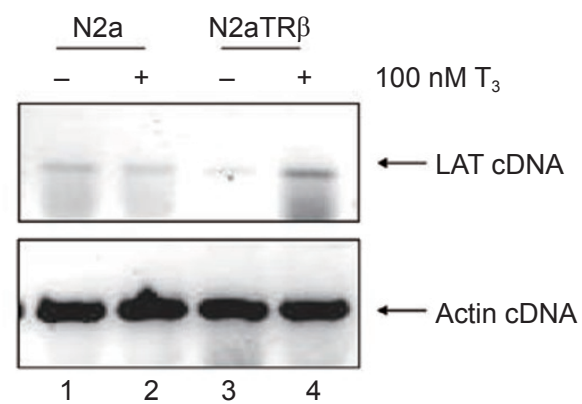

B

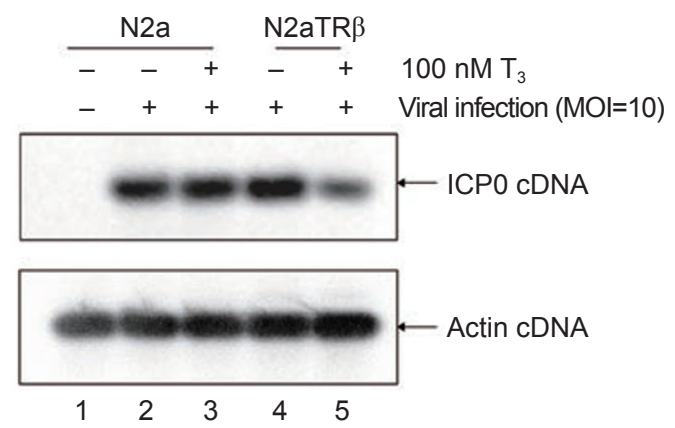

C

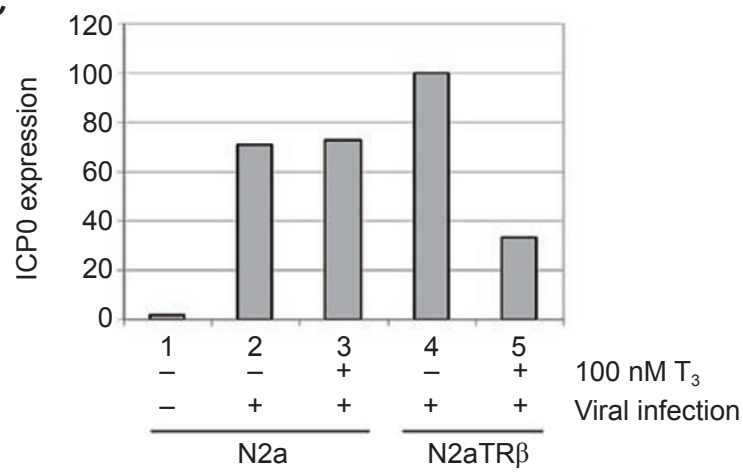

Figure 3 Regulation of LAT and ICPO by TR and $T_{3}$ during infection. (A) N2a and N2aTR $\beta$ cells were infected with wild-type virus $17 \mathrm{Syn}^{+}$at $\mathrm{MOI}=10$ with the addition of $50 \mu \mathrm{g} / \mathrm{ml}$ cycloheximide to prevent the $\alpha$-transactivation. The total RNA was purified at $8 \mathrm{hpi}$ and subjected to RT-PCR using primers against LAT. Actin primers were used as controls. (B) The RNA samples from A were used for RT-PCR assays to assess ICP0 transcription. DIG-dNTP was included in the reaction for quantitative purpose. (C) The chemiluminescence signal of samples from $\mathbf{B}$ was captured by GENEgnome camera from Syngene (Fredrick, $M D)$. The quantitative analyses were measured by Syngene's molecular biology software GeneTools.

onstrated that liganded TR up-regulated LAT expression and down-regulated ICP0 during infection.

TR $\beta$ was recruited to LAT TRE and CTCF was enriched to the same region in the presence of $T_{3}$

Direct interaction of TR and TRE was addressed by electrophoretic mobility shift assay (EMSA). Labeled oligonucleotides (oligos) were incubated with cell extract of N2a and no shifted bands were observed (Figure 4A, lanes 1-8). Labeled LAT TRE oligos generated shifted band in the presence of N2aTR $\beta$ extract (Figure 4A, lane 12 ), and this band was abolished by unlabeled oligo competition (Figure 4A, lane 13). This protein-oligo shift pattern was also observed for the positive control Xenopus TRE (xTRE) (Figure 4A, lanes 14, 15, 16). Mutant LAT TRE (from GGGAGA to GGGATT) failed to produce similar band shift (Figure 4A, lanes 9 and 10), indicating the importance of the sequence context. Collectively, these results demonstrate the in vitro interaction of TR/ LAT TRE.

The anti-TR $\beta$ antibody (Ab) was used for chromatin immunoprecipitation (ChIP) to confirm the in vivo binding of receptor to the LAT TREs. The transfection and ChIP assays showed that TR $\beta$ was recruited to the LAT TRE regardless of the ligand status (Figure 4B). In addition, this region was previously described as a chromatin insulator-like element [22]. The potential for TR $\beta$ to modulate the binding of CTCF was addressed by ChIP using anti-CTCF Ab. The results demonstrated that liganded TR $\beta$ increased the binding of CTCF to the element (Figure 4B). To address the specificity of interaction, ChIP-TR $\beta$ and ChIP-CTCF were performed using primers away from the target regions (primers against ICP4 ORF and ICP0 promoter, respectively). These negative controls produced no signal (Figure 4C), demonstrating that TR specifically interacted with LAT TREs and that the liganded TR $\beta$ could facilitate the recruitment of CTCF to the HSV-1 chromatin insulator.

Participation of histone modification and chromatin remodeling in the gene regulation of LAT

TR modulates histone modification and recruitment of chromatin remodeling complex at the TRE-containing promoters for gene regulation [34-38]. It is likely that liganded TR regulated LAT expression through these mechanisms. This hypothesis was investigated by ChIP using corresponding Abs. The LAT promoter was found to be hyperacetylated in the presence of TR and $\mathrm{T}_{3}$ (Figure $5 \mathrm{~A}$, compare 1 and 2). In addition, chromatin remodeling complex, BRG1, was enriched at the LAT promoter by liganded TR (Figure 5A, compare lanes 1 and 2) but not at the ICP0 promoter (Figure 5A, lanes 3-6), suggesting that the BRG1 complex may participate in the $\mathrm{T}_{3} /$ TR-mediated regulation. To confirm the role of BRG1 in HSV-1 gene expression, BRG1 over-expression vector pBRG1 and its dominant-negative mutant pK785R were transfected with pSG28 in N2aTR $\beta$ cells. RT-PCR assays showed that the overexpression of BRG1 had little ef- 
A
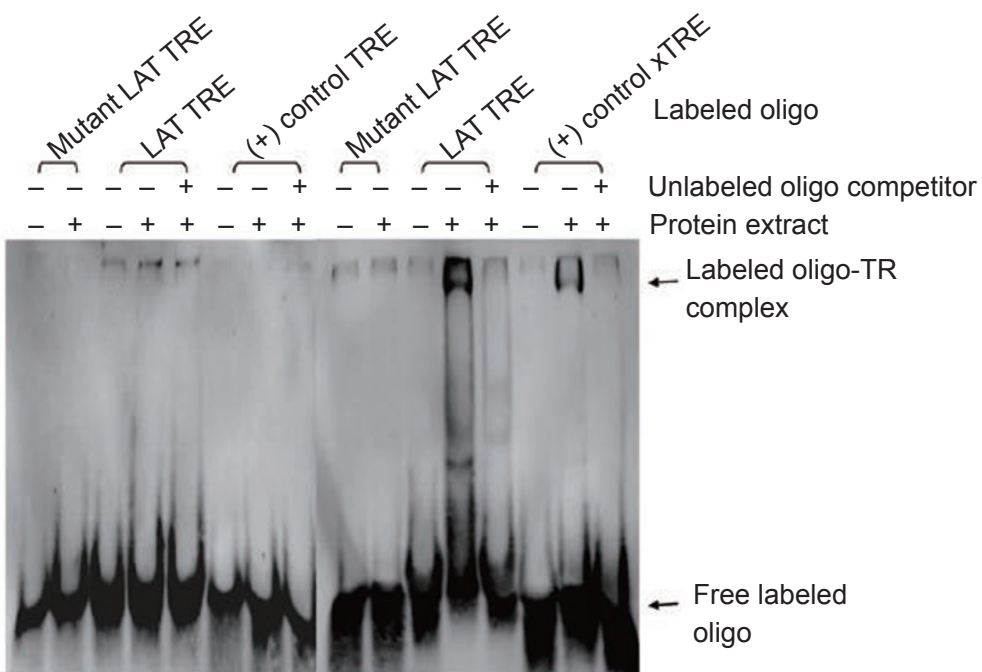

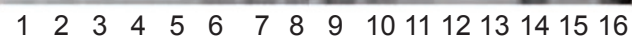

Cell extract-N2a Cell extract-N2aTR $\beta$

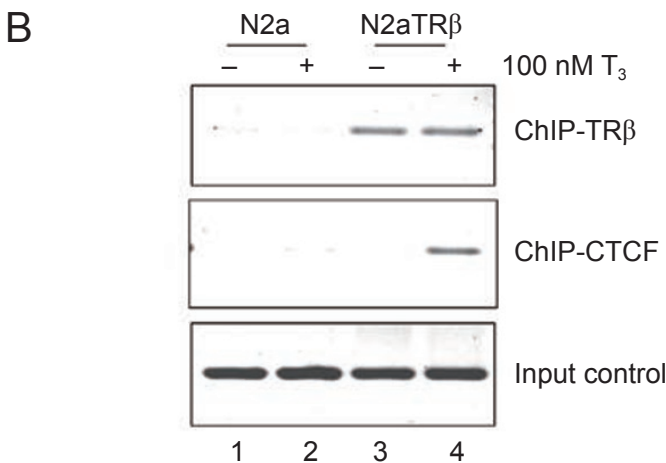

C

Primers: ICP4 ORF

Primers: ICPO promoter
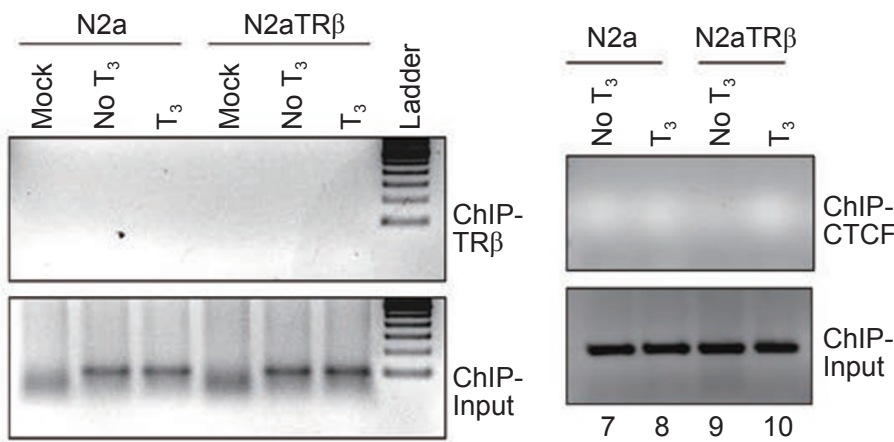

$\begin{array}{llllll}1 & 2 & 3 & 4 & 5 & 6\end{array}$

Figure 4 TR bound to LAT TRE and facilitated CTCF enrichment to LAT RE-1 in the presence of T3. (A) EMSA using extract from N2a (lanes 1-8) and N2aTR $\beta$ (lanes 9-16). Labeled oligos of LAT TRE, mutant TRE, and positive control Xenopus TRE (xTRE) were used for analyses. Free labeled oligo and labeled oligo-TR complex were marked. Note that extract of N2aTR $\beta$, but not N2a, produced a shifted band while using labeled LAT TRE oligo (lane 12) and positive control (lane 15). Mutant oligo failed to generate the pattern (lane 10). (B) Cells were transfected with pSG28 and treated with or without $\mathrm{T}_{3}$ for $48 \mathrm{~h}$. The cells were then subjected to ChIP using Abs against TR $\beta$ or CTCF for immunoprecipitation. The recruitment of TR $\beta$ or CTCF was analyzed by PCR. IgG controls showed no signal, indicating the specificity of the assays (data not shown). (C) The binding specificity of TR and CTCF to LAT TRE was further analyzed by ChIP using primers against other regions (ICP4 ORF and ICP0 promoter, respectively). Lanes 1-6: ChIP-TR $\beta$ using primers against ICP4 ORF; lanes 7-10: ChIP-CTCF using primers against ICP0 promoter. 
A

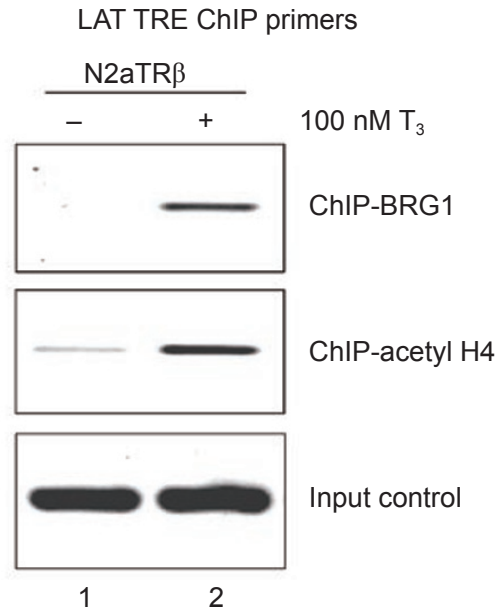

ICPO promoter primers
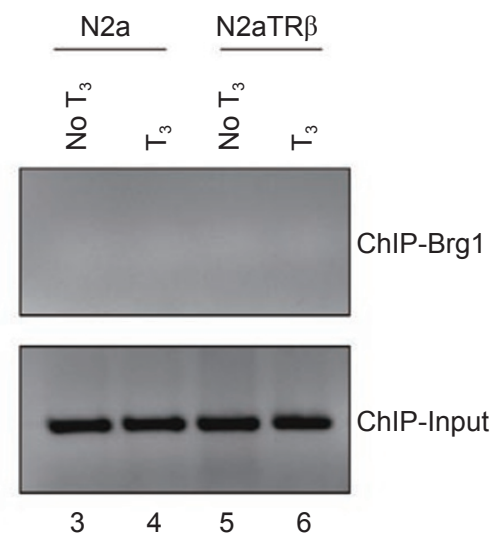

B

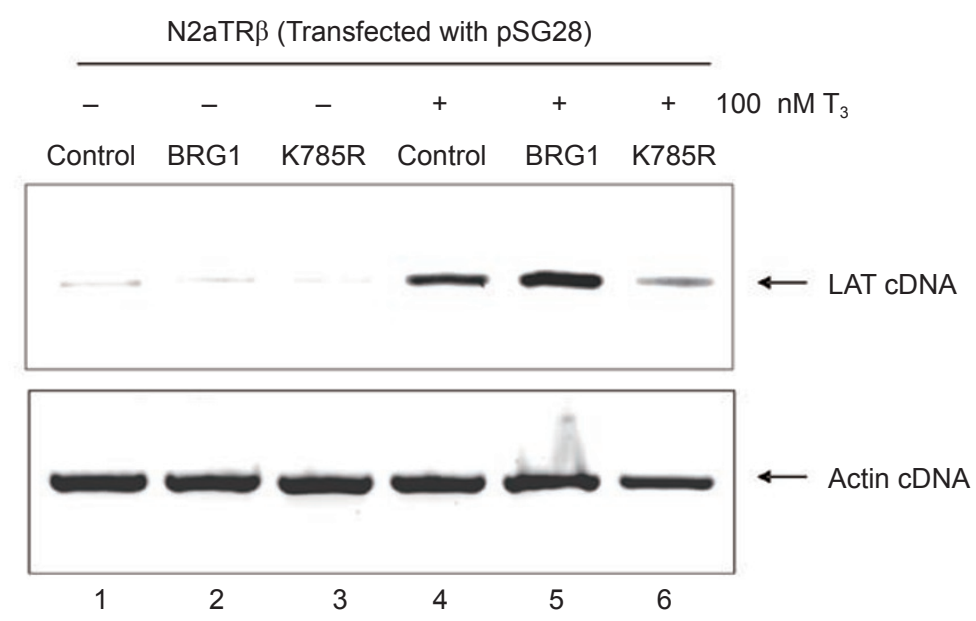

Figure 5 Participation of chromatin remodeling and histone acetylation in regulation of LAT transcription by liganded TR. (A) ChIP was performed using anti-BRG1 and anti-acetyl-histone H4 Ab followed by PCR using specific primers. IgG controls showed no signal, indicating the specificity of the assays (data not shown). Lanes 1 and 2: LAT TRE ChIP primers; lanes 3-6: ICP0 promoter primers. (B) Cells were cotransfected with pSG28 and with or without expression vectors of BRG1 or K785R specified in the figure. After $48 \mathrm{~h}$, the transfected cells were subjected to RNA isolation and RT-PCR assays using primers recognizing LAT transcript. Actin primers were used as controls.

fect on LAT expression in the absence of TR but further increased the TR-mediated LAT transcription in the presence of $\mathrm{T}_{3}$ (Figure 5B, compare lanes 1-5). The dominant-negative mutant $\mathrm{K} 785 \mathrm{R}$, on the contrary, abolished the activation by liganded TR (Figure 5B, lane 6). These results suggest that histone modification and chromatin remodeling both contribute to the $\mathrm{T}_{3} / \mathrm{TR}$-mediated LAT regulation.

Histone H4 hypoacetylation at the ICP0 promoter caused by liganded TR in the presence of LAT TRE

The BRG1 remodeling complex appeared to participate in the LAT regulation, but it did not elicit a regula- tory effect on ICP0 since overexpression of BRG1 and K786R exhibited no impact on ICP0 expression (data not shown). Nonetheless, the ICP0 promoter was hypoacetylated in the presence of liganded TR, since the ChIP assays showed that histone $\mathrm{H} 4$ acetylation was reduced at the ICP0 promoter in the presence of TR and $\mathrm{T}_{3}$ (Figure $6 \mathrm{~A})$. This $\mathrm{TR} / \mathrm{T}_{3}$-mediated hypoacetylation required the presence of LAT TREs, evidenced by the fact that the lack of LAT TRE led to no hypoacetylation at the ICP0 promoter (Figure 6B). Collectively, these results suggest that liganded TR induced hypoacetylation at the ICP0 promoter and it is likely the result of chromatin insulation mediated via LAT TREs. 
A
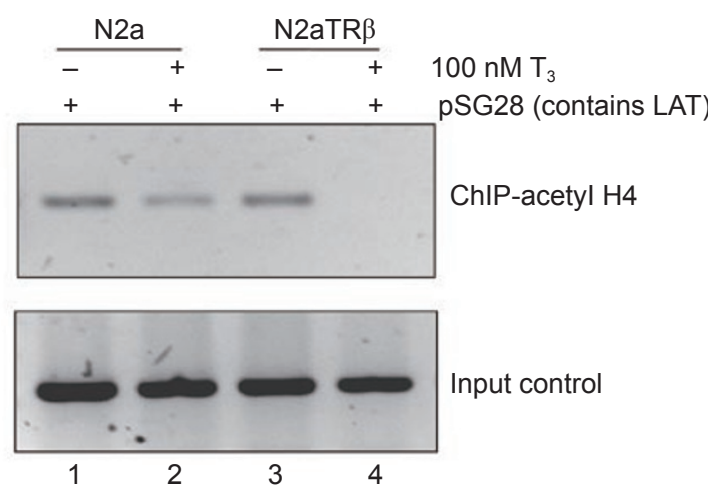

B

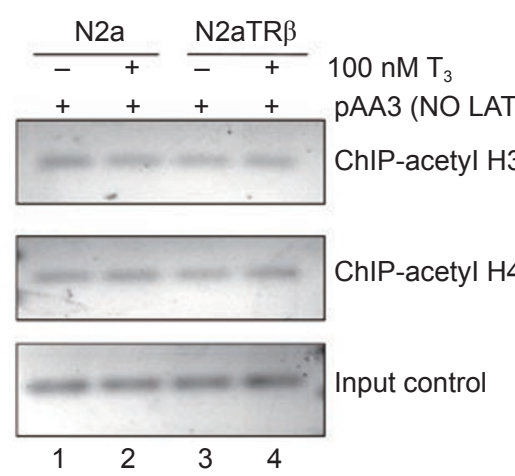

Figure $6 \mathrm{~T}_{3} / \mathrm{TR}$-mediated ICP0 promoter histone modification required the presence of LAT TRE. (A) Cells were transfected with pSG28 (containing LAT TRE) and incubated with or without $\mathrm{T}_{3}$ for $48 \mathrm{~h}$ followed by ChIP with anti-acetyl-histone $\mathrm{H} 4 \mathrm{Ab}$. The IP chromatin solution was purified and the resulting DNA was amplified by primers recognizing ICPO promoter. (B) Parallel experiments to Figure $5 \mathrm{~A}$ using plasmid pAA3 (containing no LAT TRE) were carried out with the anti-acetyl-histone $\mathrm{H} 4 \mathrm{Ab}$ as well as acetyl-histone $\mathrm{H} 3 \mathrm{Ab}$ for IP. The histone modification was analyzed by the same ICPO primers.

$T_{3}$ washout derepressed ICPO and increased infectious virus release

To investigate the effect of $\mathrm{T}_{3}$ on TR-mediated regulation during HSV-1 replication and the release of infectious virus, N2a and N2aTR $\beta$ cells were pretreated with $\mathrm{T}_{3}$ for 5 days followed by infection at MOI of 1 with or without $\mathrm{T}_{3}$ washout (Figure 7A). RT-PCR assays showed that the expression of ICP0 was de-repressed upon washout of $\mathrm{T}_{3}$ at 48 hpi in N2aTR $\beta$ but not N2a cells (Figure $7 \mathrm{~B})$. The plaque assays indicated that the $\mathrm{T}_{3}$ washout increased the virus release by more than 60 -fold compared to the cells being treated with $\mathrm{T}_{3}$ in N2aTR $\beta$ (Figure 7C). There was no change in $\mathrm{N} 2 \mathrm{a}$ cells upon $\mathrm{T}_{3}$ removal (Figure 7C). These results suggest that subtraction of hormone reversed the ICP0 repression and this viral protein may play an active role during viral replication in resting cells, leading to enhanced viral gene expression and virus release.

\section{Discussion}

This study focused on the roles of TR and its ligand $\mathrm{T}_{3}$ in the regulation of HSV-1 gene expression in neuronal cells. Our data showed that the transcription factor TR utilized its ligand to elicit differential regulatory effect on HSV-1 genes, LAT and ICP0, in neuronal cells. The results, for the first time, showed that liganded TR bound LAT TRE and activated LAT promoter via histone modification as well as BRG1-mediated chromatin remodeling. In addition, liganded TR mediated down-regulation of ICP0, at least in part, by histone hypoacetylation which requires the LAT TRE. The current model using $\mathrm{T}_{3}$-treated N2aTR $\beta$ cells was particularly useful for initial in vitro studies since N2aTR $\beta$ cells were differentiated after $\mathrm{T}_{3}$ incubation, mimicking the sensory neurons in vivo. Investigations using animal models are underway and the preliminary data indicated that $\mathrm{T}_{3}$ treatment decreased and delayed the virus release in TG explant assays, supporting the hypothesis that $\mathrm{T}_{3}$ participated in the regulation of HSV-1 latency and reactivation (data not shown).

The BRG1 complex disrupts histone-DNA interactions and has been implicated in the regulation of transcriptional activation [39]. The role of the BRG1 chromatin remodeling complex in gene regulation via nuclear hormone receptors was shown to involve modification of the nucleosomal structure to allow transcriptional activation or repression [40]. In vitro assays with several nuclear hormone receptors, including the estrogen receptor and the glucocorticoid receptor, have shown that BRG1 can modify the pattern of nucleosomal positioning and aid local chromatin structure disruption within preassembled nucleosomal display [41]. Our results showed that BRG1 was recruited to the LAT promoter by liganded TR and its dominant-negative mutant eliminated the TRmediated LAT activation. BRG1 contributes to HSV-1 IE gene expression through the VP16-HCF-Octl complex [42]. Intact VP16 complexes may not be able to enter the nucleus of sensory neurons but BRG1, on the other hand, is present in neurons. It is likely that BRG1 is recruited to $\mathrm{HSV}-1$ regulatory sequences via $\mathrm{T}_{3}$ and TR to regulate LAT transcription. More experiments are necessary to analyze the enrichment of this chromatin remodeling complex at the HSV-1 genome in neurons.

The functions of LAT and its role in ICP0 expression during HSV latency and reactivation are elusive. In neuronal cells, LAT was shown to reduce viral gene expression and replication during productive infection [43]. In 
A

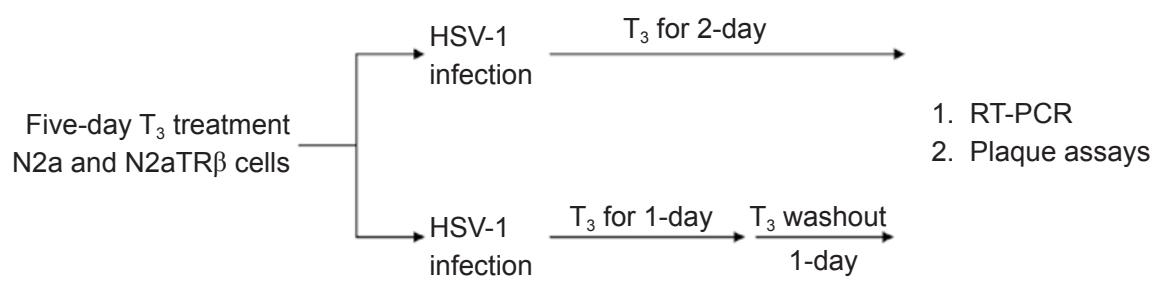

B

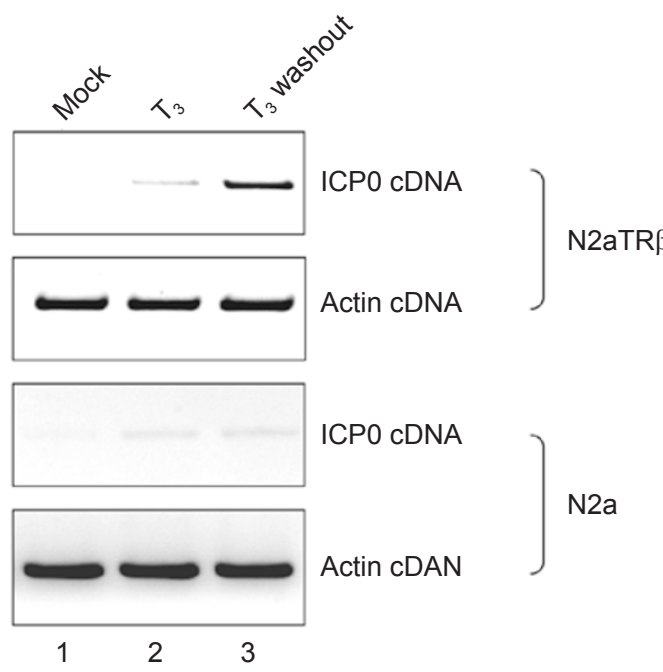

C

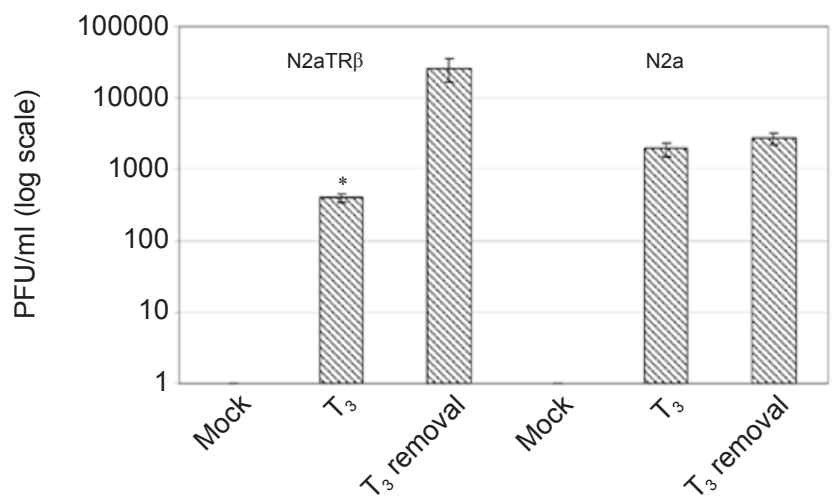

Figure 7 Long-term $\mathrm{T}_{3}$ treatment prevented the ICP0 expression and the washout of $\mathrm{T}_{3}$ derepressed the ICP0 expression and increased virus release. (A) Cells were pretreated with $T_{3}$ for 5 days and subsequently infected with 17 Syn ${ }^{+}$-EGFP viruses at $\mathrm{MOI}$ of 1 with $\mathrm{T}_{3}$. The washout was carried out a day after the infection and the assays were performed at $48 \mathrm{hpi}$. (B) Total RNA was isolated at $48 \mathrm{hpi}$ and subjected to RT-PCR assays using ICPO primers to study TR-mediated regulation. (C) The media of infected cells were collected at $48 \mathrm{hpi}$ and subjected to plaque assays using CV1 cells to investigate the release of infectious viruses. The $P$-value measured by a Student's paired $t$-test with a two-tailed distribution was 0.0015 , indicating a significant increase.

vivo, LAT mutant virus showed enhanced gene expression in sensory neurons during lytic and latent infection [44]. A more recent report showed that LAT augments the transcription of several lytic genes during the latent stage in rabbits, possibly by chromatin modifications [45]. Chromatin insulator motifs were identified within the LAT regulatory regions $[22,24]$. Our results showed that TR could bind to the LAT TREs and facilitate the 
enrichment of the chromatin insulator-binding protein CTCF to the region in the presence of $\mathrm{T}_{3}$. Under the same condition, liganded TR caused hypoacetylation of the ICP0 promoter. TREs and CTCF motifs were often adjacent and possessed overlapping functions [46-48]. In addition, ICP22 intron contains reiterative repeats similar in position and composition to the reiterative elements but different in their actual sequences. It is likely that the liganded TR facilitates the binding of CTCF to the elements or directly recruits CTCF to the sites, thus forming a boundary element (between LAT TRE/RE-1 and ICP22 intron) to regulate gene expression. Studies using mutant viruses with specific deletions are underway to investigate the effects of $\mathrm{T}_{3} / \mathrm{TR}$-mediated chromatin changes on viral gene expression and silencing in animal models.

Our results further indicated that $\mathrm{TR}$ and $\mathrm{T}_{3}$ repressed HSV-1 TK expression and modulated viral replication/release in neuronal cells (unpublished data). TK is required to provide dNTP for viral replication in resting cells such as neurons, and viral replication is required for efficient $\alpha$ and $\beta$ expression in neurons during reactivation [49]. TK was further suggested to initiate $\alpha$ transcription and subsequent replication during reactivation [50], and the mutant virus lacking TK exhibited greatly reduced $\alpha$ and $\beta$ expression during reactivation, suggesting the importance of TK [51]. Based on these results, $\mathrm{T}_{3}$ and TR may have additional regulating roles in controlling HSV-1 replication and gene expression during latency/reactivation.

Together, our studies suggest that thyroid hormone and its receptor are able to recruit different cofactors to control critical transcription of various HSV-1 genes in neuronal cells, which may have implications in regulating latency and reactivation. More experiments, especially those with mutant viruses in cell culture and animal models, are necessary to identify key mechanisms involved in HSV-1 latency and viral reactivation through the $\mathrm{TR} / \mathrm{T}_{3}$-mediated regulations.

\section{Materials and Methods}

Viruses, cell lines, and culture conditions

HSV-1 strain $17 \mathrm{Syn}^{+}$was obtained from Dr James Hill's labo- ratory at Louisiana State University Health Science Center (New Orleans, LA, USA). The N2a cell line was purchased from the American Type Culture Collection (ATCC). N2aTR $\beta$ cell is a gift from Dr Robert Denver (University of Michigan, Ann Arbor, MI, USA) and maintained in DMEM/F12 supplemented with $10 \%$ charcoal-treated fetal bovine serum. All cells were grown in an incubator at $37{ }^{\circ} \mathrm{C}$ with $5 \% \mathrm{CO}_{2} . \mathrm{T}_{3}$ was purchased from Sigma (St Louis, MO, USA).

\section{Plasmids}

Plasmid pSG28 contains HSV-1 sequence from 106785 to 131 534. It covers the LAT TRE and open reading frames such as ICP4, LAT, ICP0, etc. Plasmid pAA3 possesses the HSV-1 sequence from 122713 to 134 792. It contains partial deletion of LAT and does not have LAT TRE. Plasmid phMGFP (Promega, cat no. E6421) was used as transfection efficiency control. Plasmid pBRG1 containing the complete ORF of BRG1 was a gift from Dr Keji Zhao (NHLBI, NIH). The plasmid pK785R, another gift from Dr Zhao, contains a mutation in the BRG1 ORF with amino acid change from $\mathrm{K}$ to $\mathrm{R}$ at position 785 .

\section{Transfection}

Nucleofector II (cat no. AAD-1001S) from Amaxa (Gaithersburg, MD, USA) was used for high efficiency of transfection. The protocol was essentially described by the manufacturer. The experiments were performed using Kit V (cat no. VCA-1003) and the protocol number was T-024.

\section{Antibodies}

The Abs used for ChIP are listed in Table 1. The dilution was according to the manufacturer's suggestions.

\section{RT-PCR}

Total RNA from cells was isolated by Trizol reagent (Invitrogen, Carlsbad, CA, USA). RT-PCRs were performed using Superscript One-Step RT-PCR (Invitrogen) with $0.2 \mu \mathrm{g}$ of total RNA and correspondent primers. Their sequences are shown as follows: Actin: 5'-ATT CCT ATG TGG GCG ACG AG-3' and 5'-TGG ATA GCA ACG TAC ATG GC-3'; LAT: 5'-CGG CGA CAT CCT CCC CCT AAG $\mathrm{C}-3^{\prime}$ and 5'-GAC AGA CGA ACG AAA CGT TCC G -3'; ICP0: 5'-TTC GGT CTC CGC CTG AGA GT-3' and 5'-GAC CCT CCA GCC GCA TAC GA-3'. The RT-PCR reaction was carried out at $45{ }^{\circ} \mathrm{C}$ for $20 \mathrm{~min}$ followed by 25 cycles of $94{ }^{\circ} \mathrm{C}$ for $30 \mathrm{~s}$, $57{ }^{\circ} \mathrm{C}$ for $30 \mathrm{~s}$, and $68{ }^{\circ} \mathrm{C}$ for $30 \mathrm{~s}$. The RT-PCR products were analyzed using $2 \%$ agarose gel electrophoresis. The results were documented by Kodak Gel-Logic 100 imaging system. To increase the sensitivity of ICP0 assays, DIG-dNTP was included in the reaction for quantitative purpose. The gel was transferred to a nylon

Table 1 The sources of antibodies

\begin{tabular}{llll}
\hline Antibody & Species & Clonality & Source \\
\hline TR $\beta 1$ & Rabbit & Polyclonal & Millipore/Upstate (cat no. 06-539) \\
CTCF & Rabbit & Polyclonal & Millipore/Upstate (cat no. 07-729) \\
BRG-1 & Rabbit & Polyclonal & Abcam (cat no. ab4081) \\
Acetyl-histone H3 & Rabbit & Polyclonal & Millipore/Upstate (cat no. 17-245) \\
Acetyl-histone H4 & Rabbit & Polyclonal & Millipore/Upstate (cat no. 06-866) \\
\hline
\end{tabular}


membrane, developed by CDP-star, and measured by Syngene G:BOX lightly cooled CCD camera and molecular biology software GeneTools described previously [27].

\section{Electrophoretic mobility shift assay}

EMSA was performed using a DIG Gel shift Kit 2nd generation (Roche applied science, Indianapolis, IN, USA) essentially as described in the manufacturer's protocol. Briefly, single-stranded oligos for LAT TRE: 5'-GGG GAG AGG GGA GAG GGG GGGAGA GGG GAG AG-3'; xTRE (positive control): 5'-GAT CGC AGG TCA TTT CAG GAC AGC-3' [52]; Mutant TRE: 5'-GGG GAG AGG GGA TTG GGG GGG ATT GGG GAG AG-3' and their complementary oligos were synthesized (Invitrogen, San Diego, CA, USA) and annealed $\left(95^{\circ} \mathrm{C}\right.$ for $10 \mathrm{~min}$ followed by slow cooling) to make double-stranded (ds) oligos. The cell extracts of $\mathrm{N} 2 \mathrm{a}$ or N2aTR $\beta$ were isolated by first washing the cells twice with cold PBS followed by lysis with NP40 buffer (Invitrogen, cat no. FNN0021) containing protease inhibitor and PMSF. The ds oligos were terminally labeled with non-radioactive DIG-11-ddUTP by terminal transferase and incubated with the cell extracts of N2a or N2aTR $\beta$ for 30 min at room temperature. For competition, 10× of unlabeled oligos were added to the labeled oligos for specificity analysis. The samples were electrophoresed on a 6\% DNA Retardation Gel (Invitrogen) at $70 \mathrm{~V}$ for $1 \mathrm{~h}$ followed by electrophoretic transfer (Novex X Cell II Blot module) to positive-charged nylon membrane (Roche). The DIG-labeled oligos were visualized by an enzymatic immunoassay using anti-digoxigenin-alkaline phosphatase, Fab-fragments, and chemiluminiscent substrate CSPD (Roche). The chemiluminiscent signal was captured using GeneGenome HR imaging system (Syngene).

\section{Chromatin immunoprecipitation}

The protocol was described previously [27]. The sequences of PCR primers: ICP0 (promoter) ChIP: 5'-TAA TGG GGT TCT TTG GGG GAC ACC-3' and 5'-TGC AAA TGC GAC CAG ACT GTC-3'; LAT ChIP: 5'-GCT ACG CCT TCG GGA ATG G-3' and 5'-AGA GGG GAG CCA GTT AGA TTG C-3'; ICP4 ORF: 5'CGA CAC GGA TCC ACG ACC C-3' and 5'-GAT CCC CCT CCC GCG CTT CGT CCG-3'. The location of LAT ChIP primers (117 017-117 165) is immediately upstream of LAT TRE (117 161-117 334) since LAT TRE is highly GC rich and not suitable for PCR (Figure 1B).

\section{$T_{3}$ removal assays}

$\mathrm{N} 2 \mathrm{a}$ and N2aTR $\beta$ cells were plated on a six-well plate with $40-50 \%$ confluency with the addition of $\mathrm{T}_{3}(10 \mathrm{nM})$ into the media refreshed daily. On day 6, viral infection was performed for $1 \mathrm{~h}$ at MOI of 1 . The inoculum was then removed and the cells were washed twice with $1 \mathrm{ml}$ of PBS followed by the addition of $1 \mathrm{ml}$ of fresh media containing $\mathrm{T}_{3}$ for 1 day. The $T_{3}$ incubation was stopped after 1 day by removing the medium completely and washing the cells twice with PBS. New medium was added in each well with or without $\mathrm{T}_{3}$. The media were collected at 48 hpi and subjected to RT-PCR and plaque assays. The paradigm was shown in Figure 7A.

\section{Acknowledgments}

This project is supported by NIH/NCRR P20RR016456. We thank Dr Keji Zhao from NHLBI/NIH for BRG1 expression vectors. We are grateful for the 17Syn ${ }^{+}$-EGFP virus from Dr James Hill of LSUHSC in New Orleans, LA, USA. Our gratitude also goes to Dr Robert Denver (Ann Arbor, MI, USA) for the N2aTR $\beta$ cell line.

\section{References}

1 Goldenberg D, Mador N, Ball MJ, Panet A, Steiner I. The abundant latency-associated transcripts of herpes simplex virus type 1 are bound to polyribosomes in cultured neuronal cells and during latent infection in mouse trigeminal ganglia. J Virol 1997; 71:2897-2904.

2 Javier RT, Stevens JG, Dissette VB, Wagner EK. A herpes simplex virus transcript abundant in latently infected neurons is dispensable for establishment of the latent state. Virology 1988; 166:254-257.

3 Jones C. Herpes simplex virus type 1 and bovine herpesvirus 1 latency. Clin Microbiol Rev 2003; 16:79-95.

4 Wu TT, Su YH, Block TM, Taylor JM. Evidence that two latency-associated transcripts of herpes simplex virus type 1 are nonlinear. J Virol 1996; 70:5962-5967.

5 Cook ML, Thompson RL, Stevens JG. A herpes simplex virus mutant is temperature sensitive for reactivation from the latent state: evidence for selective restriction in neuronal cells. Virology 1986; 155:293-296.

6 Ecob-Prince M, Hassan K. Reactivation of latent herpes simplex virus from explanted dorsal root ganglia. $J$ Gen Virol 1994; 75 (Pt 8):2017-2028.

7 Kriesel JD, Ricigliano J, Spruance SL, Garza HH Jr, Hill JM. Neuronal reactivation of herpes simplex virus may involve interleukin-6. J Neurovirol 1997; 3:441-448.

8 Sawtell NM, Thompson RL. Comparison of herpes simplex virus reactivation in ganglia in vivo and in explants demonstrates quantitative and qualitative differences. J Virol 2004; 78:7784-7794.

9 Koelle DM, Corey L. Herpes simplex: insights on pathogenesis and possible vaccines. Annu Rev Med 2008; 59:381-395.

10 Vider-Shalit T, Fishbain V, Raffaeli S, Louzoun Y. Phasedependent immune evasion of herpesviruses. J Virol 2007; 81:9536-9545.

11 Bystricka M, Russ G. Immunity in latent herpes simplex virus infection. Acta Virol 2005; 49:159-167.

12 Morrison LA, Knipe DM. Mechanisms of immunization with a replication-defective mutant of herpes simplex virus 1 . Virology 1996; 220:402-413.

13 Cui C, Griffiths A, Li G, et al. Prediction and identification of herpes simplex virus 1-encoded microRNAs. J Virol 2006; 80:5499-5508.

14 Block T, Barney S, Masonis J, et al. Long term herpes simplex virus type 1 infection of nerve growth factor-treated PC12 cells. J Gen Virol 1994; 75 (Pt 9):2481-2487.

15 Moxley MJ, Block TM, Liu HC, et al. Herpes simplex virus type 1 infection prevents detachment of nerve growth factordifferentiated PC12 cells in culture. J Gen Virol 2002; $83(\mathrm{Pt}$ 7):1591-1600.

$16 \mathrm{Su}$ YH, Moxley M, Kejariwal R, et al. The HSV 1 genome in quiescently infected NGF differentiated PC12 cells cannot be stimulated by HSV superinfection. J Neurovirol 2000; 6:341- 
349.

$17 \mathrm{Su} \mathrm{YH}$, Moxley MJ, Ng AK, et al. Stability and circularization of herpes simplex virus type 1 genomes in quiescently infected PC12 cultures. J Gen Virol 2002; 83 (Pt 12):29432950.

18 Garza HH Jr, Hill JM. Effect of a beta-adrenergic antagonist, propranolol, on induced HSV-1 ocular recurrence in latently infected rabbits. Curr Eye Res 1997; 16:453-458.

19 Hardwicke MA, Schaffer PA. Differential effects of nerve growth factor and dexamethasone on herpes simplex virus type 1 oriL- and oriS-dependent DNA replication in PC12 cells. J Virol 1997; 71:3580-3587.

20 Marquart M, Bhattacharjee P, Zheng X, et al. Ocular reactivation phenotype of HSV-1 strain F(MP)E, a corticosteroidsensitive strain. Curr Eye Res 2003; 26:205-209.

21 Noisakran S, Halford WP, Veress L, Carr DJ. Role of the hypothalamic pituitary adrenal axis and IL-6 in stress-induced reactivation of latent herpes simplex virus type 1. J Immunol 1998; 160:5441-5447.

22 Amelio AL, McAnany PK, Bloom DC. A chromatin insulatorlike element in the herpes simplex virus type 1 latencyassociated transcript region binds CCCTC-binding factor and displays enhancer-blocking and silencing activities. $J$ Virol 2006; 80:2358-2368.

23 Bedadala GR, Pinnoji RC, Hsia SC. Early growth response gene 1 (Egr-1) regulates HSV-1 ICP4 and ICP22 gene expression. Cell Res 2007; 17:1-10.

24 Chen Q, Lin L, Smith S, et al. A CTCF-dependent chromatin boundary element exists between the LAT and ICP0 promoters in the HSV-1 genome. J Virol 2007 Jan 31. doi: 10.1128/ JVI.0244-06

25 Kubat NJ, Amelio AL, Giordani NV, Bloom DC. The herpes simplex virus type 1 latency-associated transcript (LAT) enhancer/rcr is hyperacetylated during latency independently of LAT transcription. J Virol 2004; 78:12508-12518.

26 Kubat NJ, Tran RK, McAnany P, Bloom DC. Specific histone tail modification and not DNA methylation is a determinant of herpes simplex virus type 1 latent gene expression. $J$ Virol 2004; 78:1139-1149.

27 Pinnoji RC, Bedadala GR, George B, et al. Repressor element-1 silencing transcription factor/neuronal restrictive silencer factor (REST/NRSF) can regulate HSV-1 immediateearly transcription via histone modification. Virol J 2007; 4:56.

28 Knipe DM, Cliffe A. Chromatin control of herpes simplex virus lytic and latent infection. Nat Rev Microbiol 2008; 6:211221.

29 Deme D, Pommier J, Nunez J. Kinetics of thyroglobulin iodination and of hormone synthesis catalysed by thyroid peroxidase. Role of iodide in the coupling reaction. Eur J Biochem 1976; 70:435-440.

30 Papavasiliou SS, Martial JA, Latham KR, Baxter JD. Thyroid hormonelike actions of 3,3',5'-L-triiodothyronine nad 3,3'diiodothyronine. J Clin Invest 1977; 60:1230-1239.

31 Lazar MA. Thyroid hormone receptors: multiple forms, multiple possibilities. Endocr Rev 1993; 14:184-193.

32 Tsai MJ, O'Malley BW. Molecular mechanisms of action of steroid/thyroid receptor superfamily members. Annu Rev Biochem 1994; 63:451-486.

33 Yen PM. Physiological and molecular basis of thyroid hor- mone action. Physiol Rev 2001; 81:1097-1142.

34 Hsia SC, Shi YB. Chromatin disruption and histone acetylation in regulation of the human immunodeficiency virus type 1 long terminal repeat by thyroid hormone receptor. Mol Cell Biol 2002; 22:4043-4052.

35 Hsia SC, Tomita A, Obata K, et al. Role of chromatin disruption and histone acetylation in thyroid hormone receptor action: implications in the regulation of HIV-1 LTR. Histol Histopathol 2003; 18:323-331.

36 Huang ZQ, Li J, Sachs LM, Cole PA, Wong J. A role for cofactor-cofactor and cofactor-histone interactions in targeting p300, SWI/SNF and Mediator for transcription. EMBO J 2003; 22:2146-2155.

37 Lee KC, Li J, Cole PA, Wong J, Kraus WL. Transcriptional activation by thyroid hormone receptor-beta involves chromatin remodeling, histone acetylation, and synergistic stimulation by $\mathrm{p} 300$ and steroid receptor coactivators. Mol Endocrinol 2003; 17:908-922.

38 Wallberg AE, Yamamura S, Malik S, Spiegelman BM, Roeder RG. Coordination of p300-mediated chromatin remodeling and TRAP/mediator function through coactivator PGC-1alpha. Mol Cell 2003; 12:1137-1149.

39 Kadam S, Emerson BM. Transcriptional specificity of human SWI/SNF BRG1 and BRM chromatin remodeling complexes. Mol Cell 2003; 11:377-389.

40 Yoshinaga SK, Peterson CL, Herskowitz I, Yamamoto KR. Roles of SWI1, SWI2, and SWI3 proteins for transcriptional enhancement by steroid receptors. Science 1992; 258:15981604.

41 Wang S, Zhang B, Faller DV. BRG1/BRM and prohibitin are required for growth suppression by estrogen antagonists. EMBO J 2004; 23:2293-2303.

42 Herrera FJ, Triezenberg SJ. VP16-dependent association of chromatin-modifying coactivators and underrepresentation of histones at immediate-early gene promoters during herpes simplex virus infection. $J$ Virol 2004; 78:9689-9696.

43 Mador N, Goldenberg D, Cohen O, Panet A, Steiner I. Herpes simplex virus type 1 latency-associated transcripts suppress viral replication and reduce immediate-early gene mRNA levels in a neuronal cell line. $J$ Virol 1998; 72:5067-5075.

44 Garber DA, Schaffer PA, Knipe DM. A LAT-associated function reduces productive-cycle gene expression during acute infection of murine sensory neurons with herpes simplex virus type 1. J Virol 1997; 71:5885-5893.

45 Giordani NV, Neumann DM, Kwiatkowski DL, et al. During Hsv-1 infection of Rabbits, the ability to express the lat increases latent-phase transcription of lytic genes. J Virol 2008; 82:6056-6060.

46 Awad TA, Bigler J, Ulmer JE, et al. Negative transcriptional regulation mediated by thyroid hormone response element 144 requires binding of the multivalent factor CTCF to a novel target DNA sequence. J Biol Chem 1999; 274:2709227098.

47 Burke LJ, Zhang R, Lutz M, Renkawitz R. The thyroid hormone receptor and the insulator protein CTCF: two different factors with overlapping functions. J Steroid Biochem Mol Biol 2002; 83:49-57.

48 Lutz M, Burke LJ, LeFevre P, et al. Thyroid hormone-regulated enhancer blocking: cooperation of CTCF and thyroid 
hormone receptor. $E M B O J$ 2003; 22:1579-1587.

49 Nichol PF, Chang JY, Johnson EM Jr, Olivo PD. Herpes simplex virus gene expression in neurons: viral DNA synthesis is a critical regulatory event in the branch point between the lytic and latent pathways. J Virol 1996; 70:5476-5486.

50 Tal-Singer R, Peng C, Ponce De Leon M, et al. Interaction of herpes simplex virus glycoprotein $\mathrm{gC}$ with mammalian cell surface molecules. J Virol 1995; 69:4471-4483.
51 Kosz-Vnenchak M, Jacobson J, Coen DM, Knipe DM. Evidence for a novel regulatory pathway for herpes simplex virus gene expression in trigeminal ganglion neurons. J Virol 1993; 67:5383-5393.

52 Hsia SC, Wang H, Shi YB. Involvement of chromatin and histone acetylation in the regulation of HIV-LTR by thyroid hormone receptor. Cell Res 2001; 11:8-16. 